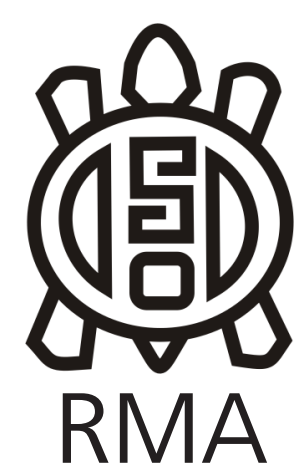

Dossier

\title{
Explotación y circulación de rocas de grano fino oscuras tipo Potrok Aike: el análisis de dos conjuntos líticos de Patagonia extremo sur continental (Argentina)
}

\author{
Exploitation and circulation of fine-grained dark rocks type Potrok Aike: \\ the analysis of two lithic assemblages of southernmost continental \\ Patagonia (Argentina) \\ Natalia A. Cirigliano*, M. Cecilia Pallo** y Judith E. Charlin*** \\ * CONICET, Instituto Multidisciplinario de Historia y Ciencias Humanas. \\ E-mail: naticirigliano@hotmail.com \\ ** CONICET, Instituto Patagónico de Ciencias Sociales y Humanas y Facultad de \\ Filosofía y Letras, Universidad de Buenos Aires. E-mail: ceciliapallo@gmail.com \\ *** CONICET, Instituto Multidisciplinario de Historia y Ciencias Humanas y Facultad de \\ Filosofía y Letras, Universidad de Buenos Aires. E-mail: judith.charlin@gmail.com
}

\begin{abstract}
Resumen
El objetivo de este trabajo es analizar la abundancia, características tecno-morfológicas y la intensidad de uso de los artefactos confeccionados con Rocas de Grano fino Oscuras tipo Potrok Aike (RGFO PKA) en dos muestras de superficie recuperadas en diferentes sectores de Patagonia extremo sur continental. Estos conjuntos fueron obtenidos en Laguna La Argentina, una fuente secundaria de RGFO PKA ubicada en el centro del campo volcánico Pali Aike, y Laguna Cóndor, situada en la zona de los Morros (al noroeste del campo volcánico Pali Aike), donde esta materia prima no se encontró disponible naturalmente. Los resultados alcanzados muestran variaciones en la explotación de la variedad PKA, que se encuentran en relación con la disponibilidad de esta roca y el rango de acción de los grupos de cazadores-recolectores en estos espacios durante el Holoceno tardío.
\end{abstract}

Palabras clave: rocas de grano fino oscuras tipo Potrok Aike; explotación; cazadores-recolectores; zona de los morros; campo volcánico Pali Aike.

\begin{abstract}
The aim of this paper is to analyse the abundance, techno-morphological characteristics and the exploitation of artifacts made from Fine-Grained Dark Rocks Potrok Aike type (RGFO PKA) of two surface samples recovered at different sector of southernmost part of the Patagonia. These assemblages were obtained from Laguna La Argentina, a secondary source of RGFO PKA located in the center of the Pali Aike volcanic field, and Laguna Cóndor, situated in the zone of the Morros (northwest of the Pali Aike volcanic field), where this raw material is not naturally available. The achieved results show variations in the exploitation of the PKA variety, which are related to the availability of this rock and the hunter-gatherer home range in theses spaces during the late Holocene.
\end{abstract}

Keywords: Fine-Grained Dark Rocks Potrok Aike type; explotation; hunter-gatherer; zone of the morros; Pali Aike volcanic field.

\section{Introducción}

El campo volcánico Pali Aike (en adelante CVPA) y la zona de los morros se localizan en el extremo sur de Patagonia continental. En estos espacios la estructura de los recursos líticos se encuentra caracterizada principalmente por la existencia de dacita de variados colores y un conjunto de rocas oscuras de grano fino de diverso origen -volcánico, sedimentario y metamórfico- denominadas RGFO (Charlin 2005, 2009, 2012). En el grupo de las RGFO y a partir de la observación microscópica de cortes delgados, se distinguió una variedad volcánica que fue denominada "tipo Potrok Aike" (PKA). La variedad PKA es una dacita vítrea de muy buena calidad para la talla, de coloración negra oscura y brillosa, que presenta una pasta con fenocristales de tamaños pequeños y corteza vesicular (Charlin y D'Orazio 2015; Charlin y Pallo 2013). Esta variedad de roca registra una disponibilidad relativamente abundante en algunas lagunas del interfluvio GallegosChico, centro del CVPA (Figura 1) (Borrazzo et al. 2019; Charlin y D'Orazio 2015; Charlin y Pallo 2013). El tipo PKA se reconoció también en baja frecuencia en el río El Zurdo -al sur de su confluencia con el río Gallegos-, el curso medio e inferior del río Gallegos y en Punta Loyola -costa Atlántica- (Charlin y D'Orazio 2015; Charlin y Pallo 2013, 2015). A excepción de los escasos ejemplares 
recuperados en el río El Zurdo, la RGFO PKA no fue identificada en los espacios occidentales y noroccidentales al CVPA. En dichos sectores los muestreos de materias primas líticas exhibieron principalmente dacitas de diversas coloraciones y RGFO sedimentarias -lutitas y fangolitas-. En menor frecuencia también se registran RGFO volcánicas -andesitas, basaltos-, riolitas, cuarzos, jaspes, diabasas, tobas y otras rocas indiferenciadas de génesis variable (Charlin 2012; Charlin et al. 2011). Por lo tanto, a escala regional, se puede reconocer al sector central del CVPA como una gran área de captación de RGFO PKA (Figura 1).

En vista de que las RGFO PKA se encuentran localizadas en fuentes secundarias (sensu Luedtke 1979) de un sector particular del CVPA, en el interfluvio Gallegos-Chico, y que fueron ampliamente utilizadas en el pasado para la manufactura de artefactos en Patagonia extremo sur (Borrazzo et al. 2019; Charlin y D'Orazio 2015; Charlin y Pallo 2013), se pretendió conocer cómo los grupos cazadores-recolectores que ocuparon dicho espacio efectuaron su explotación en el pasado. En este sentido, se estudiaron y compararon los artefactos confeccionados con RGFO PKA de dos conjunto líticos distantes entre sí en ca. $113 \mathrm{~km}$ (lineal). Se trata de los conjuntos recuperados en Laguna La Argentina, fuente secundaria de nódulos de la variedad PKA, ubicada en el interfluvio Gallegos-Chico -centro del CVPA-, y Laguna Cóndor, localizada en la llamada zona de los Morros, al noroeste del campo de lava, donde este tipo de roca no se encontró disponible naturalmente (Figura 1). Cabe destacar que las materias primas identificadas en ambas lagunas fueron efectivamente explotadas por los cazadores-recolectores, al menos durante el Holoceno tardío (Borrazzo et al. 2019;
Charlin 2012; Charlin et al. 2011).

\section{Modelo y expectativas}

Dado que Laguna La Argentina presenta evidencias de explotación de RGFO PKA y se encuentra dentro del rango de acción estimado para las poblaciones que ocuparon el CVPA y áreas adyacentes durante el Holoceno tardío (e.g. Borrero y Charlin 2010, Charlin 2009), el acceso a la fuente se podría haber llevado a cabo de manera directa. Por tal motivo, para el estudio del conjunto lítico y su relación con el área de captación de RGFO PKA, se utilizó el modelo cuantitativo de distribución espacial propuesto por Renfrew (1977) que considera la abundancia de artefactos según la distancia al área de aprovisionamiento lítico. En caso de acceso directo, el modelo sostiene un decrecimiento en la frecuencia de artefactos a medida que aumenta la distancia a la fuente, lo que conforma una curva de caída o fall off. Asimismo, se siguió la propuesta de Khun (2004) para evaluar el grado de reducción de los artefactos. La disponibilidad variable de RGFO PKA en el área permitió generar expectativas arqueológicas diversas para los distintos espacios investigados. En los conjuntos artefactuales de Laguna La Argentina, al tratarse de una fuente secundaria de RGFO PKA, se espera encontrar alta frecuencia de artefactos elaborados con esta materia prima, estadios iniciales e intermedios de talla (presencia de núcleos y desechos de talla) y baja/media intensidad de uso de artefactos. En cambio, las expectativas arqueológicas en casos en los que se verifique el transporte de RGFO PKA hacia espacios donde ésta no se encuentra disponible, como en Laguna Cóndor, podría involucrar estrategias de economía de materia prima (Odell 1996), baja frecuencia de artefactos
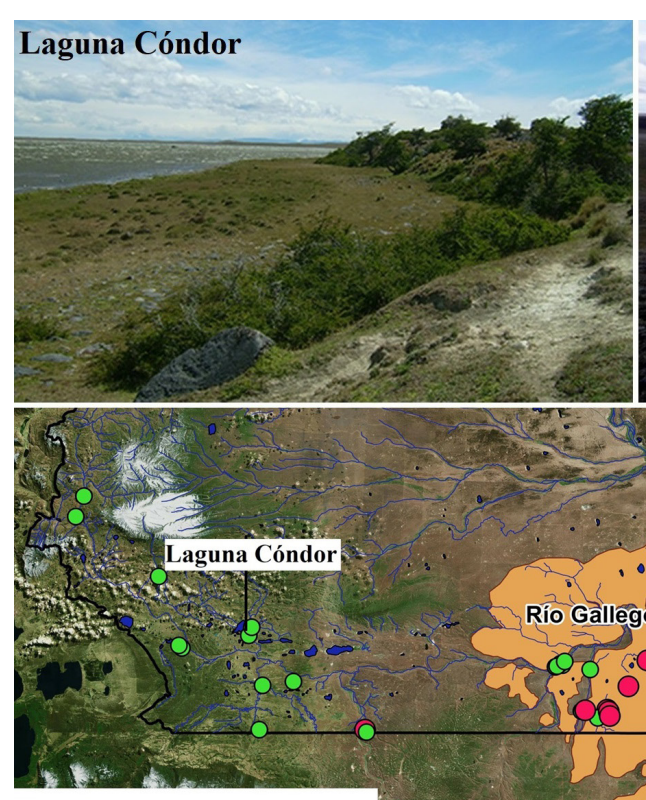

Muestreos de rocas:

O Ausencia de RGFO PKA Presencia de RGFO PKA $\square$ Campo Volcánico Pali Aike $\square$ Límite Argentina/Chile
Laguna La Argentina
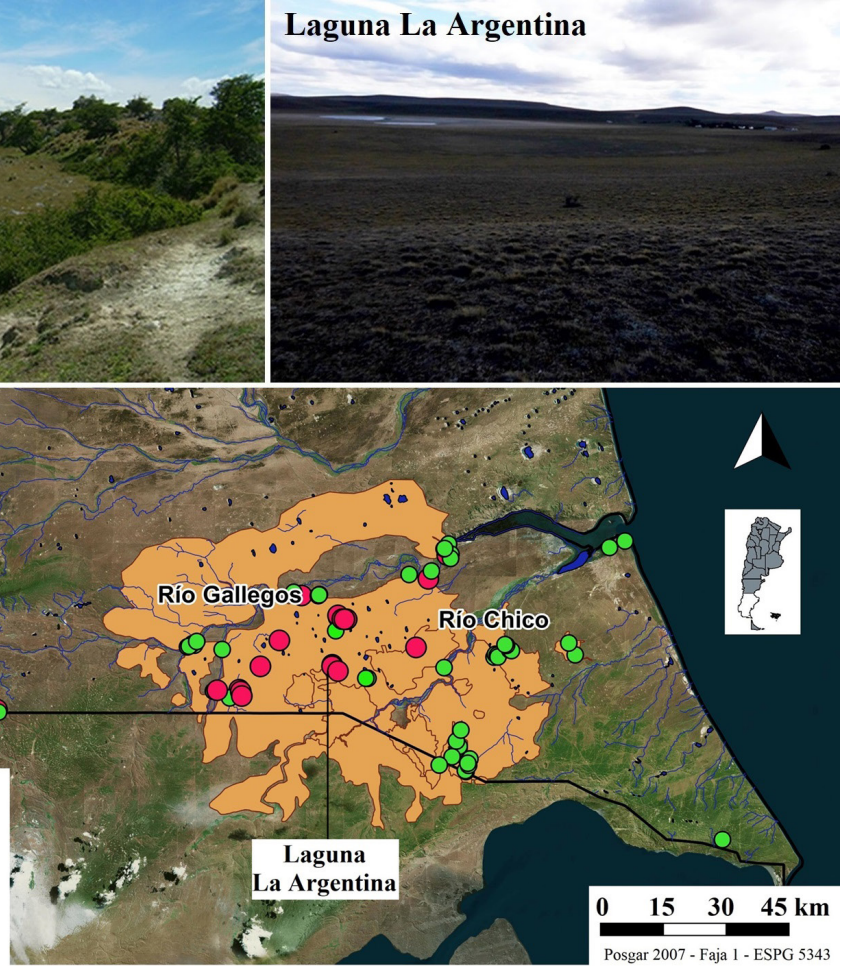

Figura 1. Sectores bajo estudio.

Figure 1. Location of studied sectors. 
en RGFO PKA (Renfrew 1977) y alta intensidad de uso de los mismos (Franco 2007).

\section{Metodología}

Inicialmente se caracterizó la disponibilidad de RGFO PKA en la fuente secundaria Laguna La Argentina según su abundancia relativa, calidad (sensu Aragón y Franco 1997), morfología (Graham and Midgley 2000, 2003; Sneed y Folk 1958), tamaño y volumen. Allí, se efectuaron dos muestreos de rocas sobre la margen norte de la laguna, cada uno realizado por una persona durante cinco minutos siguiendo la metodología propuesta por Franco y Borrero (1999). Dado que en Laguna Cóndor no se recuperaron nódulos de RGFO PKA en los muestreos realizados, se remite a Charlin (2012) y Charlin et al. (2011) para una caracterización de la fuente (ver Supra). Por otro lado, se analizaron los conjuntos líticos de Laguna La Argentina $(n=65)$ y Laguna Cóndor $(n=442)$ procedentes de recolecciones de superficie y a cielo abierto que cubrieron un área de $26.000 \mathrm{~m}^{2}$ y 30.000 $\mathrm{m}^{2}$ respectivamente. Los materiales fueron recuperados a través de cuadrículas de recolección en cicatrices de erosión en Laguna Cóndor (años 2008 y 2011) y de transectas en Laguna La Argentina (año 2017). La recolección de los artefactos líticos en este último espacio coincidió con la localización de los muestreos de materias primas.

El análisis tecno-morfológico se limitó a los artefactos elaborados con RGFO PKA siguiendo los lineamientos propuestos por Aschero (1975/1983) y Flehengeimer et al. (1995). Asimismo, se buscó conocer la intensidad de explotación de los núcleos y desechos de talla utilizando el índice propuesto por Ingbar et al. (1989), adaptado por Charlin (2009) para el caso de los núcleos. Para ello se contabilizó la cantidad de negativos de lascado en la cara dorsal del desecho en relación con el área ${ }^{1}$, exceptuando las lascas de reactivación, y el número de extracciones del núcleo sobre el volumen ${ }^{2}$ del mismo. No se estimó la intensidad de uso de los instrumentos debido a su escasez en Laguna Cóndor. Por último, mediante el uso del programa PAST (Hammer 2017) se utilizaron tests de hipótesis para la comparación de las medias de las variables analizadas en ambos conjuntos artefactuales (t de Student y $\mathrm{t}$ de Welch, según la naturaleza de las varianzas, Hammer 1999-2018).

\section{Resultados}

\section{La fuente secundaria Laguna La Argentina}

En conjunto, los dos muestreos de rocas realizados arrojaron una abundancia relativa de $95,36 \%$ nódulos de la variedad PKA ( $n=42 / 47)$. De éstos, el 95,24\% presentó muy buena aptitud para la talla, mientras que el resto se

1 CantidaAd de negativos de lascado en la cara dorsal/área (largo por ancho) de la lasca por 100.

${ }^{2}$ Cantidad de extracciones en el núcleo/volumen por 1000. Aquí el resultado se multiplica por 1000 para reducir el número de decimales obtenidos. dividió en calidades buenas (2,38\%) y malas (2,38\%). Todos los ejemplares registran corteza. En general, los tamaños de los guijarros son medianos y pequeños. Las dimensiones promedio son 75,5 $\mathrm{mm}$ para el eje mayor $\left(\mathrm{s}^{3}=26,92 \mathrm{~mm}\right), 55,27 \mathrm{~mm}$ para el eje menor $(\mathrm{s}=20,18$ $\mathrm{mm}$ ) y $37,42 \mathrm{~mm}$ para el espesor $(\mathrm{s}=15,44 \mathrm{~mm})$. El volumen medio es 215.310,5 $\mathrm{mm}^{3}\left(\mathrm{~s}=247.249,8 \mathrm{~mm}^{3}\right)$. Se identificaron nódulos con formas elongadas-proladas $(52,38 \%)$, compactas $(45,24 \%)$ y muy aplanadaselongadas (2,38\%). De estos datos se desprende que la alta frecuencia de nódulos con formas compactas o esféricas, sin plataformas naturales adecuadas (Shelley 1993), sumado al alto porcentaje de guijarros con volúmenes reducidos, podría tener implicancias en el uso de la técnica bipolar y en el tipo de elemento seleccionado en caso de transporte hacia otros espacios (e.g. nódulo).

Los artefactos elaborados con RGFO PKA de Laguna La Argentina y Laguna Cóndor

En Laguna La Argentina se recuperó un total de 65 artefactos líticos. Entre los ejemplares registrados, el $84,61 \%(n=55)$ se encontró confeccionado con RGFO PKA. Se trata de 11 núcleos, 34 desechos de talla, nueve instrumentos y un artefacto con rastros complementarios. Respecto a los núcleos (20\%), más de la mitad son bipolares (54,54\%). El resto son prismáticos parciales unidireccionales $(18,18 \%)$, bifaciales $(18,18 \%)$ y con lascados aislados $(9,1 \%)$. Todos los ejemplares se encuentran enteros. La longitud máxima y mínima es de $70 \mathrm{~mm}$ y $32 \mathrm{~mm}$ respectivamente. El volumen máximo es de $234.995 \mathrm{~mm}^{3}$, mientras que el mínimo es de $10.450 \mathrm{~mm}^{3}$. Las densidades de extracciones por $\mathrm{mm}^{3}$ están comprendidas entre 0,05 y 0,57. Todos los núcleos registran reserva de corteza, entre $10 \%$ y $85 \%$. En cuanto a los desechos de talla $(61,82 \%)$, se contabilizaron entre 0 y 1,3 extracciones $/ \mathrm{mm}^{2}$. Si consideramos únicamente los ejemplares con talón, incluyendo los astillados $(n=25)$, se registra un predominio de lascas angulares (40\%), seguidas de las bipolares (20\%), secundarias (12\%), primarias (12\%), indiferenciadas (12\%) y en tableta de núcleo (4\%). Los talones registrados son astillados $(32 \%)$, corticales $(28 \%)$, lisos $(28 \%)$, lisos-corticales (4\%), diedros (4\%) y filiformes (4\%). La longitud máxima y mínima de los ejemplares enteros es de $63 \mathrm{~mm}$ y 15 $\mathrm{mm}$ respectivamente. La reserva de corteza varía entre $0 \%$ y $100 \%$, existiendo un alto porcentaje de desechos con esta característica $(86,87 \%)$. Entre los instrumentos $(16,36 \%)$, el filo más representado es el de raspador $(26,67 \%)$, seguido de raedera $(13,33 \%)$, retoque en bisel asimétrico oblicuo o RBO $(13,33 \%)$, biface $(13,33 \%)$ y no diferenciado (13,33\%). También se registran filos de cuchillo $(6,67 \%)$, denticulado $(6,67 \%)$ y cortante $(6,67 \%)$. La formatización se efectuó sobre lascas $(77,78 \%)$ y formas base indeterminadas (22,22\%). Los instrumentos presentan filos compuestos $(44,45 \%)$, simples $(33,33 \%)$ y bifaciales $(22,22 \%)$. El $88,88 \%$ de los ejemplares formatizados registra restos de corteza, entre $5 \%$ y $85 \%$, y el $66,67 \%$ se encuentra entero. Por último, se reconoció

3 Desviación estándar 
un fragmento distal de lasca indiferenciada con rastros complementarios y reserva de corteza.

Una situación diferente se presenta en Laguna Cóndor, donde se obtuvieron 442 artefactos. Entre las piezas registradas, sólo 26 ejemplares se encontraron elaborados con RGFO PKA (5,88\%). Se identificaron dos núcleos (7,69\%), 22 desechos de talla (84,62\%) y dos instrumentos $(7,69 \%)$. Los núcleos se encuentran enteros, uno es bipolar y el otro prismático parcial unidireccional. Las longitudes de ambos son $57 \mathrm{~mm}$ y $26 \mathrm{~mm}$, mientras que los volúmenes, $235.125 \mathrm{~mm}^{3}$ y $4.368 \mathrm{~mm}^{3}$ respectivamente. Se registran 0,03 y 2,06 extracciones/ $\mathrm{mm}^{3}$ y restos de corteza en ambos núcleos (5\% y 50\%). De los 22 desechos de talla, sólo 10 tienen talón: seis lascas angulares (60\%), tres lascas de reactivación directa (30\%) y una lasca con dorso natural (10\%). Se reconocieron tres talones astillados (30\%), dos lisos (20\%), dos filiformes (20\%), uno diedro (10\%), uno facetado (10\%) y uno puntiforme (10\%). La longitud máxima de los desechos enteros es de 35,5 mm, mientras que la mínima es de 5,5 $\mathrm{mm}$. Más de la mitad de los ejemplares enteros registra corteza $(57,14 \%)$, cubriendo hasta un $35 \%$ del total de las piezas. Se contabilizaron entre 0,11 y 7,02 extracciones/ $\mathrm{mm}^{2}$. En cuanto a los artefactos formatizados se reconocieron dos puntas de proyectil sobre forma base indeterminada. Éstas se asemejan morfológicamente a las adscriptas por Bird (1988) al período IV. Una de las puntas se encuentra entera y reactivada. Se trata de un ejemplar con pedúnculo diferenciado y hombros. La otra, se halla representada por un fragmento de limbo. Ninguna exhibe reserva de corteza.

\section{La explotación de RGFO PKA}

La comparación entre los conjuntos líticos de ambos sectores muestra diferencias en la intensidad de explotación de RGFO PKA. En primer lugar, esto queda evidenciado por la mayor frecuencia relativa de la variedad PKA registrada en el conjunto artefactual de Laguna La Argentina (84,61\%) en oposición al de Laguna Cóndor (5,88\%). Específicamente, entre los núcleos de Laguna La Argentina se reconoce mayor proporción de RGFO PKA y variedad de tipos y tamaños, lo que es consistente con lo esperado para una fuente de aprovisionamiento lítico. La variabilidad en el porcentaje de reserva de corteza sugiere diversidad en la explotación de núcleos, aunque domina la baja intensidad de uso dado el cálculo de extracciones por volumen. La alta frecuencia de núcleos bipolares se puede explicar por las características morfológicas y métricas de los nódulos registrados en la fuente. Por otra parte, los volúmenes máximos de los núcleos, similares en algunos casos en Laguna La Argentina y Laguna Cóndor, se explica posiblemente por el transporte de nódulos de RGFO PKA desde las fuentes localizadas en el centro del CVPA hacia Laguna Cóndor. En este sentido, cabe mencionar que en Laguna La Argentina se registraron nódulos con volúmenes de hasta $1.090 .773 \mathrm{~mm}^{3}$. En cambio, el pequeño volumen y la alta densidad de extracciones registradas en uno de los núcleos (bipolar agotado) de Laguna Cóndor sugieren la alta explotación de esta materia prima en espacios alejados a las fuentes de abastecimiento.

En cuanto a los desechos, hay mayor riqueza de tipos $(n=6)$ en el conjunto de Laguna La Argentina que en Laguna Cóndor $(n=3)$. En el primer conjunto predominan las lascas angulares, bipolares, primarias y secundarias, mientras que en el segundo lo hacen las lascas angulares y de reactivación de instrumentos. Entre los talones del conjunto de Laguna La Argentina dominan los astillados, corticales y lisos, mientras que en los de Laguna Cóndor lo hacen los astillados, lisos y filiformes. Estos datos sugieren la ejecución de distintos estadios de talla en Laguna La Argentina, donde prevalecen los eventos iniciales. Por el contrario, en Laguna Cóndor se observa la existencia de eventos intermedios, finales y de mantenimiento de instrumentos. Cabe mencionar que la bipolaridad se reconoce en ambos espacios (núcleos y lascas bipolares, talones astillados). La comparación entre los conjuntos de desechos de talla muestra diferencias significativas en las medias de longitud ( $t=2,3115, p=<0,05$ ) y densidad de extracciones ( $\mathrm{t}$ Wech $=-2,6943, \mathrm{p}=<$ $0,05)$. En este sentido, Laguna La Argentina registra una longitud media mayor y una densidad de extracciones/ $\mathrm{mm}^{2}$ menor que Laguna Cóndor. También hay diferencias significativas en las medias que refieren a la cantidad de reserva de corteza ( $\mathrm{t}$ Welch $=4,2666, \mathrm{p}=<0,05$ ), siendo ésta menor en Laguna Cóndor (Figura 2). En líneas generales, los análisis muestran que la intensidad de uso de los artefactos recuperados en Laguna Cóndor es significativamente mayor que la de aquéllos obtenidos en Laguna La Argentina.

En cuanto a los instrumentos, Laguna La Argentina registra mayor abundancia y variabilidad de grupos tipológicos, así como ejemplares con reserva de corteza. Por el contrario, en Laguna Cóndor sólo se recuperaron puntas de proyectil fracturadas o agotadas. La heterogeneidad del conjunto instrumental y el tamaño reducido de la muestra de Laguna Cóndor no permiten comparar la intensidad de uso de esta clase artefactual entre ambos sectores.

Las tendencias observadas son las esperadas debido a que los conjuntos analizados se localizan a diferentes distancias del área de captación de RGFO PKA. Las características del conjunto lítico elaborado sobre la variedad PKA en Laguna Cóndor (e.g. baja frecuencia relativa de artefactos, escasez de instrumentos) sugieren que la adquisición de la materia prima se habría dado de manera directa. El transporte bajo la forma de nódulos o núcleos poco explotados hacia la Laguna Cóndor quedaría sustentado por las particularidades del registro lítico allí recuperado: diversidad artefactual (núcleos, desechos de talla e instrumentos), secuencia de talla completa (manufactura, uso, descarte y mantenimiento de instrumentos) y alto porcentaje de desechos de talla con reserva de corteza. La distancia entre Laguna Cóndor y la fuente de captación más cercana y previsible de RGFO PKA se calculó en ca. 88 km lineales (Laguna Potrok 

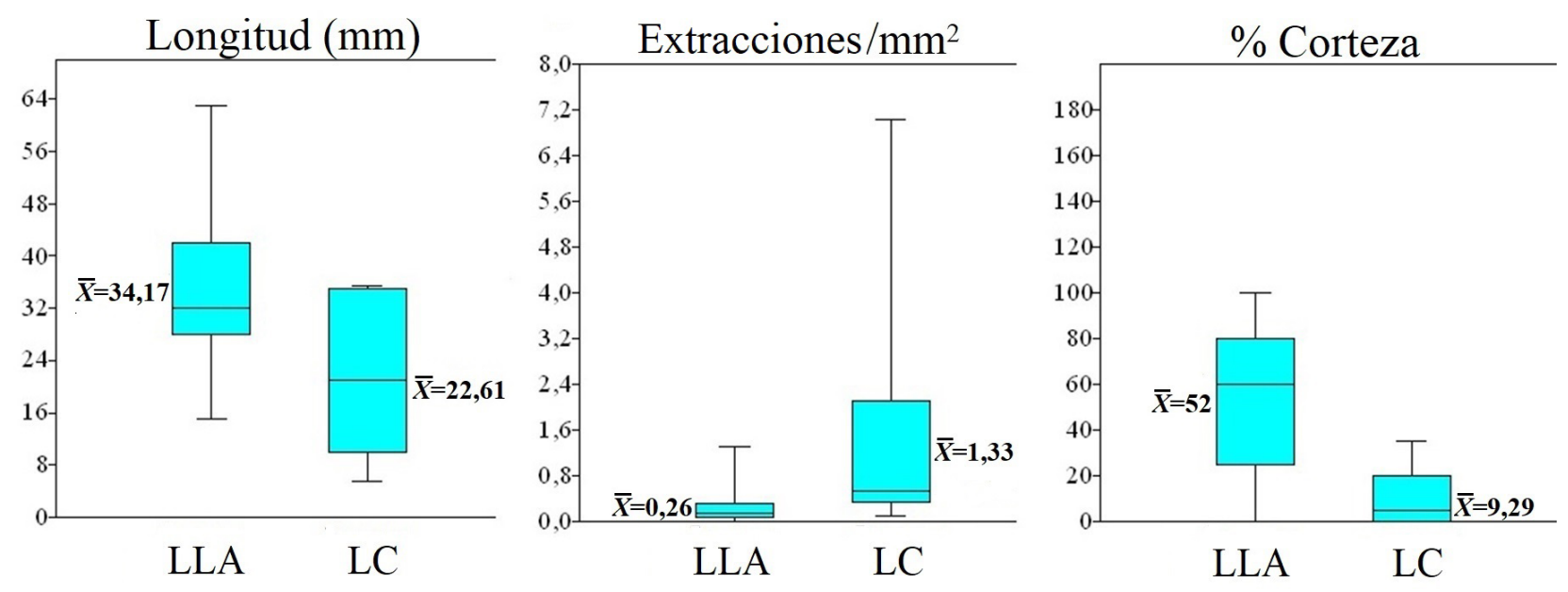

Figura 2: gráfico de cajas de las variables que arrojaron diferencias estadísticamente significativas en los test de hipótesis. Referencias: LLA (Laguna La Argentina) y LC (Laguna Cóndor).

Figure 2: boxplots of variables that showed statistically significant differences in hypothesis tests.

Aike, centro del CVPA), aunque no se puede descartar la posibilidad de recuperar nódulos de esta variedad de roca en forma impredecible y en muy baja frecuencia en el río El Zurdo (ca. 38 km en línea recta desde Laguna Cóndor).

\section{Conclusión}

El conocimiento acerca de la base regional de los recursos líticos y la forma de aprovisionamiento de rocas es un paso necesario para comprender el sistema tecnológico de los grupos humanos (Ericson 1984). En este sentido, el estudio y la descripción de la fuente secundaria Laguna La Argentina brindó información novedosa acerca de la disponibilidad y características de los nódulos de RGFO PKA, a la vez que permitió generar expectativas sobre el modo de explotación y la forma de transporte de esta variedad de roca por parte de los cazadores-recolectores en el pasado. Los resultados obtenidos a partir del análisis de los conjuntos artefactuales localizados en espacios con y sin disponibilidad natural de RGFO PKA mostraron variaciones en la explotación de esa materia prima. Éstas se encuentran en asociación con la disponibilidad y las características de los nódulos de la variedad PKA y con el rango de acción de los grupos humanos que ocuparon el CVPA durante el Holoceno tardío.

La baja frecuencia de artefactos elaborados con RGFO PKA en Laguna Cóndor y la falta de éstos en espacios localizados más al norte podrían marcar un límite al transporte de esta roca bajo la forma de nódulos y/o artefactos desde el centro del CVPA. Los resultados alcanzados apoyan lo señalado en investigaciones previas que sostienen que el espacio comprendido por Laguna Cóndor, el CVPA y las áreas inmediatas a la costa atlántica y el estrecho de Magallanes conforman un área de circulación o rango de acción poblacional. Este área se caracterizaría por la explotación de determinadas materias primas (e.g. RGFO PKA), la manufactura de lascas y por la existencia de indicadores (isótopos, bienes marinos) que muestran conexiones con sectores costeros. Por el contrario, el espacio localizado al norte de Laguna Cóndor y noroeste del CVPA, con otras evidencias arqueológicas (e.g. uso de RGFO sedimentarias, tecnología laminar), se vincularía con otra esfera de circulación humana (Borrero y Charlin 2010 y citas allí incluidas; Charlin et al. 2011; entre otros). Por lo anteriormente expuesto, los artefactos elaborados con RGFO PKA constituyen una línea de evidencia fundamental para apoyar la existencia de dos núcleos poblacionales con rangos de acción diferenciales en Patagonia extremo sur continental. Como agenda a futuro se considera relevante la exploración de los espacios intermedios; es decir entre la Laguna Cóndor y el CVPA, con el objeto de conocer la disponibilidad de los recursos líticos y el modo en que se dio la explotación de la variedad PKA en el pasado. Se espera que futuras investigaciones arrojen luz en este sentido.

Ciudad Autónoma de Buenos Aires, 6 de Junio 2019

\section{Agradecimientos}

Agradecemos los comentarios de los evaluadores anónimos que contribuyeron a mejorar este trabajo. Proyectos ANPCyT (PICT 2014-2061) y CONICET (postdoctoral); Otros proyectos: PIP-CONICET No 5676 y PICT-ANPCyT N² 2046, dirigidos por el Dr. Luis Borrero. Se agradece a las familias Clark, Fernández y Kennard por su apoyo brindado durante los trabajos de campo.

\section{Referencias citadas.}

Aragón, E. y Franco, N. (1997). Características de rocas para la talla por percusión y propiedades petrográficas. Anales del Instituto de la Patagonia 25, 187-189.

Aschero, C. (1975/1983). Ensayo para una clasificación morfológica de los artefactos líticos aplicada a estudios tipológicos-comparativos. Informes en CONICET. 
Bird, J. (1988). Travels and Archaeology in South Chile. Iowa: University of lowa Press.

Borrazzo, K., L'Heureux, L., Cirigliano, N., Ozán, I., Pallo, C., Manzi, L. (2019). Arqueología del interfluvio GallegosChico (Santa Cruz, Argentina): nuevas prospecciones. En Décimas Jornadas de Arqueología de la Patagonia (En Prensa).

Borrero, L. y Charlin, J. (2010). Arqueología del Campo Volcánico Pali Aike, Argentina. En L. Borrero y J. Charlin (Eds.), Arqueología de Pali Aike y Cabo Vírgenes (Santa Cruz, Argentina), (pp. 9-30). Buenos Aires: CONICETIMHICIHU.

Charlin, J. (2005). Utilización de materias primas líticas en el campo volcánico Pali Aike (Pcia. Santa Cruz, Argentina). Una primera aproximación a partir del análisis de los núcleos. Werken 2, 39-55.

Charlin, J. (2009). Estrategias de aprovisionamiento y utilización de las materias primas líticas en el campo volcánico Pali Aike (Prov. Santa Cruz, Argentina). Oxford, Inglaterra: Archaeopress.

Charlin, J. (2012). Materias primas líticas y uso del espacio en las nacientes del río Gallegos: el caso de laguna Cóndor (Estancia Glencross, Santa Cruz, Argentina). Magallania 40, 163-184. doi: 10.4067/S0718-22442012000100010

Charlin, J., Borrero, L. y Pallo, C. (2011). Ocupaciones humanas en el área noroccidental del río Gallegos (Prov. Santa Cruz, Argentina). En L. Borrero y K. Borrazzo Eds.), Bosques, montañas y cazadores. Investigaciones arqueológicas en Patagonia meridional, (pp. 179-210). Buenos Aires: CONICET-IMHICIHU.

Charlin, J., y D'Orazio, M. (2015). Disponibilidad de materias primas líticas en la costa nororiental del estrecho de Magallanes (Chile). Una aproximación exploratoria. Magallania 43, 133-154. doi: 10.4067/ S0718-22442015000200007

Charlin, J., y Pallo, C. (2013). Disponibilidad de materias primas líticas y uso del espacio en el interfluvio GallegosChico (Pali Aike, Santa Cruz, Argentina). En A. Zangrando et al. (Comp.), Tendencias teórico-metodológicas y casos de estudio en la arqueología de la Patagonia (pp. 307316). Buenos Aires: Altuna Impresores.

Charlin, J., y Pallo, C. (2015). Disponibilidad de rocas y costos de aprovisionamiento en el extremo austral de Patagonia meridional: integración de resultados en una escala regional. Intersecciones en Antropología - Volumen Especial 2, 125-138.

Ericson, J. (1984). Toward the analysis of lithic reduction systems. En J. Ericson y B. Purdy (eds.), Prehistoric Quarries and Lithic Production (pp. 1-19). Cambridge: Cambridge University Press.

Flegenheimer, N., Bayón, C., y Gonzalez de Bonaveri, M. (1995). Técnica simple, comportamientos complejos: la talla bipolar en la arqueología bonaerense. Relaciones de la Sociedad Argentina de Antropología XX, 81-110.

Franco, N. y L. Borrero (1999). Metodología de análisis de la estructura regional de recursos líticos. En C. Aschero et al. (Eds.), En los Tres Reinos, (pp. 27-37). Tucumán: UNT-IML.

Franco, N. (2007). Lithic Artifacts and the Information about Human Utilization of Large Areas. En P. Escola y S. Hocsman (Eds.), Artefactos Líticos, Movilidad y Funcionalidad de Sitios: Problemas y Perspectivas, Oxford: British Archaeological Reports, John and Erica Hedge Ltd.

Graham, D. y Midgley N. (2000). Graphical representation of particle shape using triangular diagrams: an Excel spreadsheet method. Earth Surface Processes and Landforms 25(13), 1473-1477.

Graham, D. y Midgley, N. (2003). Tri-plot: Ternary diagram plotting de la Universidad de Loughborough. Recuperado de https://www.lboro.ac.uk/microsites/research/physgeog/tri-plot/in dex.html

Hammer, Ø. (2017). PAST 3.15. http://folk.uio.no/ ohammer/past/

Hammer, Ø. (1999-2018). PAST Paleontological Statistics Version 3.15. Reference manual. Natural History Museum University of Oslo. Recuperado de http://folk.uio.no/ ohammer/past/past3manual.pdf

Ingbar, E., Larson, M. y Bradley, B. (1989). A nontypological approach to debitage analysis. En D. Amick y R. Mauldin (eds.), Experiments in lithic technology (pp. 117-135). Oxford: British Archaeological Report International Series 528

Instituto Geográfico Nacional de la República Argentina (2018). Capas SIG. Recuperado de http://www.ign.gob. ar/NuestrasActividades/InformacionGeoespacial/CapasSIG

Kuhn, S. (2004). Upper Paleolithic raw material economies at Ücagizli cave, Turkey. Journal of Anthropological Archaeology 23: 431-448. doi: 10.1016/j.jaa.2004.09.001

Luedtke, B. (1979). The identification of sources of chert artifacts. American Antiquity 44, 744-756.

Odell, G. (1996). Economizing behavior and the concept of "curation". En G. Odell (ed.), Stone Tools: Theoretical Insights into Human Prehistory (pp. 51-80). New York: Plenum Press.

Renfrew, C. (1977). Alternative models for exchange and spatial distribution. En T. Earle y J. Ericson (Eds.), Exchange Systems in Prehistory (pp. 71-90). New York: Academic Press.

Shelly, P. (1993). A geoarchaeological approach to the analysis of secondary lithic deposits. Geoarchaeology: An International Journal 8(1), 59-72.

Sneed, E. y Folk, R. (1958). Pebbles in the Lower Colorado River, Texas a Study in Particle Morphogenesis. The Journal of Geology 66(2), 114-150. 